\title{
The interactions between thermodynamic anomalies
}

Cite as: J. Chem. Phys. 151, 024502 (2019); https://doi.org/10.1063/1.5103242

Submitted: 25 April 2019 . Accepted: 13 June 2019 . Published Online: 10 July 2019

Domagoj Fijan (iD, and Mark Wilson (iD)

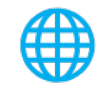

\section{ARTICLES YOU MAY BE INTERESTED IN}

Is water one liquid or two?

The Journal of Chemical Physics 150, 234503 (2019); https://doi.org/10.1063/1.5096460

Perspective: Excess-entropy scaling

The Journal of Chemical Physics 149, 210901 (2018); https://doi.org/10.1063/1.5055064

Ions' motion in water

The Journal of Chemical Physics 150, 190901 (2019); https://doi.org/10.1063/1.5090765

\section{Lock-in Amplifiers up to $600 \mathrm{MHz}$}
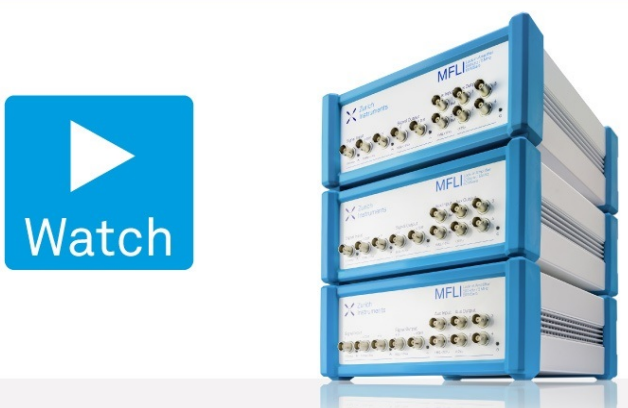

J. Chem. Phys. 151, 024502 (2019); https://doi.org/10.1063/1.5103242 


\title{
The interactions between thermodynamic anomalies
}

\author{
Cite as: J. Chem. Phys. 151, 024502 (2019); doi: 10.1063/1.5103242 \\ Submitted: 25 April 2019 - Accepted: 13 June 2019 • \\ Published Online: 10 July 2019
}

Domagoj Fijan ${ }^{1,2, a)}$ (D) and Mark Wilson'

\begin{abstract}
AFFILIATIONS
${ }^{1}$ Physical and Theoretical Chemistry Laboratory, Department of Chemistry, University of Oxford, South Parks Road, Oxford OX1 3QZ, United Kingdom

${ }^{2}$ Institute of Industrial Science, University of Tokyo, 4 Chome-6-1 Komaba, Meguro City, Tokyo 153-8508, Japan
\end{abstract}

a)domagoj.fijan@gmail.com

\begin{abstract}
The origin of and interactions between key thermodynamic anomalies are derived and analyzed, as are the interactions with the stability (or cavitation) limits. The conditions for interaction are derived from the underlying thermodynamic relations rather than using the morecommonly applied Taylor expansion method. As a result, we derive a general set of equations that govern the interactions between different lines of thermodynamic anomalies using standard manipulation of thermodynamic equations. The validity of the derivations is investigated by comparing them to numerical simulation data and previous Taylor expansion-based results. Simulations are performed using a modified Stillinger-Weber potential in which the balance of the two- and three-body interactions is varied and which serves to highlight the relationships between the various anomalies. The deeply supercooled regime is explored by employing replica exchange methods. The behavior of the anomalies is considered in terms of previously constructed thermodynamic "scenarios." Based on the newly uncovered interaction schemes, we propose a classification strategy for the thermodynamic anomalies (as first- or second-order) which could be extended to additional related anomalies.
\end{abstract}

Published under license by AIP Publishing. https://doi.org/10.1063/1.5103242

\section{INTRODUCTION}

The density maximum observed in water at $T \sim 4{ }^{\circ} \mathrm{C}$ and ambient pressure is perhaps the most well-known and well-studied example of a thermodynamic anomaly. However, water and a number of other key materials display anomalies in related properties such as the compressibilities and heat capacities. ${ }^{1-30}$ The consequences of these phenomena may be numerous and far reaching, covering areas such as the existence of life on Earth to fundamental connections to important technological processes and materials (such as silicon or phase change materials used in IT equipment).

Besides water, the next best studied system in terms of the thermodynamical anomalies is silicon. ${ }^{11,29,31-35}$ Since thermodynamic anomalies often appear in metastable regions of phase space, simulations are often used to probe these regions as they may not be easily accessible to experimental techniques. In the case of silicon, simulations are often performed using the StillingerWeber (SW) potential. ${ }^{36}$ The SW potential contains a two body
Lennard-Jones-like term and a three body term that biases the local configuration toward a certain ideal bonding angle. This potential has been thoroughly studied in terms of potential anomalous behavior for silicon ${ }^{11,28,29,31-35,37}$ and for alternative parameterizations to model related systems. ${ }^{36,38-40}$ The flexibility to simulate a wide range of anomalous systems by changing the relative strength of two and three body terms has made the SW potential a key model for studying this behavior although soft-core potentials have also been successfully employed. ${ }^{41-52}$ Although some of the work referenced above $^{29}$ employs methods to push the limits of metastability (by employing replica exchange techniques, for example), the anomalies in deeply supercooled regions of the phase space have not been reported in literature to date (in particular, in the low temperature limit). Related investigations, using the SW potential have studied the progression of the anomalous behavior as a function of the strength of the three body term by fitting the thermodynamic results to a two state model. ${ }^{32}$ However, relatively little attention has been paid to high values of the three body term (close to the point 
where the anomalous behavior disappears) and the low temperature region has not been fully explored. As a result, the SW potential is extremely convenient for studying anomalous behavior as the details of the anomalies can be systematically altered via a single (physically transparent) potential parameter (which controls the relative strength of two and three body terms).

Thermodynamic anomalies are normally displayed as loci (lines of maxima or minima of a given thermodynamic quantity, usually in the $P T$ or $V T$ planes) which may highlight distinct regions encompassing the anomalous behavior. One of the first attempts to analyze the interactions between these loci was via the stability limit conjecture (SLC), ${ }^{53,54}$ which was based on extrapolations from experimental data for water. The SLC [see Fig. 1(a)] describes the collision between the temperature of maximum density (TMD) locus and the line of liquid stability (the liquid branch of the liquid-vapor spinodal line). ${ }^{53}$ This work was expanded by Debenedetti and co-workers, focusing on the so-called tensile instability, ${ }^{5-57}$ a direct consequence of the SLC scenario, in which the TMD line collides with the spinodal. In that work, a Taylor expansion approach was utilized to analyze the specific conditions under which the TMD and liquid-vapor spinodal lines intersect. As a result, this work also predicted the disappearance of the TMD line away from the spinodal by merging with a temperature of minimum density locus (TminD). ${ }^{58}$ This merging of the two loci describing the density anomalies was first reported in simulations by Poole and co-workers, evoking a so-called second critical point scenario [see Fig. 1(b)].

Potential anomalous behavior other than that associated with the density (for example, in the heat capacity or compressibility) has received relatively little to no exposure in terms of their analytical description and potential interaction with other anomalies until the proposal of the singularity free scenario. ${ }^{4,59}$ In this work, the gradient of the TMD line when intersecting the temperature of minimum compressibility (TminC) loci was derived. The behavior of the TminC under these conditions was analyzed using the Taylor expansion method of Debenedetti. ${ }^{55}$ A similar approach was used to explain the gradients of the density anomalies and temperature of maximum heat capacity (TMH) on collision at low pressures. ${ }^{60}$ The interactions between the density, compressibility, and heat capacity anomalies has been analyzed for stretched water using a combination of acoustic measurements and simulation. $^{61}$

In this work, we derive expressions for the interaction of lines of density, compressibility, and heat capacity anomalies as well as corresponding interactions with the spinodal line and/or the stability limit. Instead of applying the widely used Taylor expansion approach, we simply manipulate thermodynamic equations. This approach yields more general relations that can be applied to any combination of anomalies (i.e., minima and maxima) and does not require additional justifications in order to neglect the importance of surviving higher order terms. However, this approach cannot probe the possible termination points of the anomaly loci (as studied by Debenedetti and D'Antonio's work ${ }^{58}$ ). We are able to derive all the collision scenarios reported from the Taylor expansion-based methods. We also show that newly reported collisions (from numerical simulations using the SW potential) also follow the same rules and we highlight these new interactions, for example, between the TMC and TminD loci and the TminH and TminC. We also show that

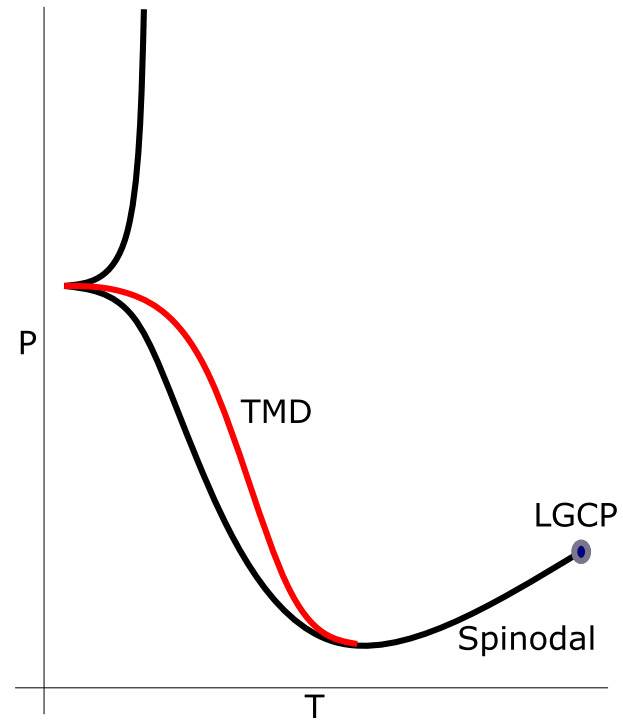

(a)

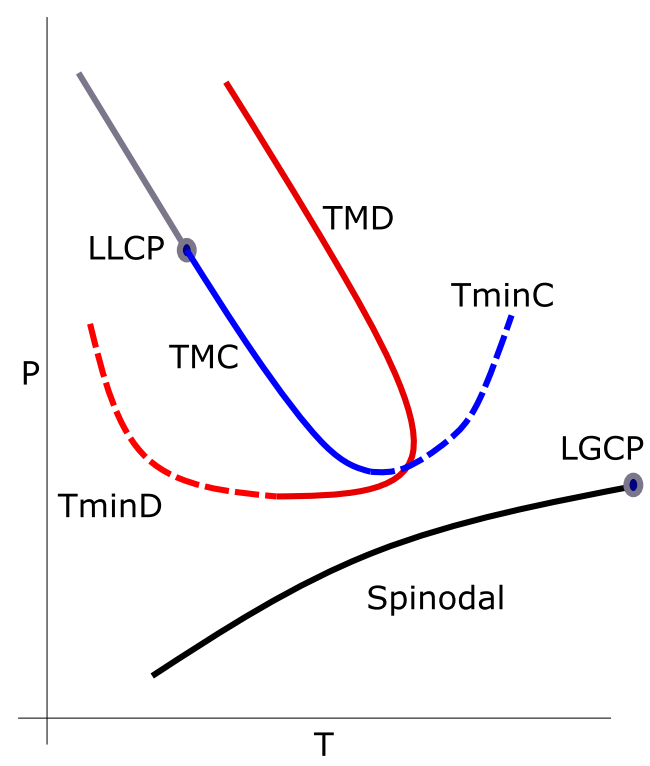

(b)

FIG. 1. Thermodynamic scenarios describing the anomalous behavior in the disordered systems. TMD and TminD refer to the temperature of maximum and minimum density lines and TMC and TminC to the temperature of maximum and minimum compressibility lines. LLCP and LGCP refer to the liquid-liquid and liquidgas critical points, respectively. Panel (a) shows the stability limit conjecture (SLC) whilst panel (b) shows the second (liquid-liquid) critical point scenario.

additional collision scenarios could exist which have not yet been observed. For example, we show that if the TMD and TminD swap places, then the TEC locus scenarios considered in Ref. 4 would have inverted TMC/TminC and TMH/TminH gradients. Furthermore, we consider a method for identifying the source of the density 
anomaly and consider the origins of the compressibility and heat capacity anomalies. We employ replica exchange molecular dynamics (REMD) simulations ${ }^{62,63}$ to help explore the deeply supercooled regime which may be rich in new interactions between thermodynamic anomalies. These results serve as a test to probe the validity of our thermodynamic derivations. Finally, we propose a classification scheme for lines of anomalous behavior according to their definition, interaction, and general behavior. These could easily be extended to other lines defined in a similar manner.

\section{THE ANOMALIES AND STABILITY LIMIT. DERIVATIONS AND INTERACTION}

In all our derivations, we consider a disordered system with constant number of particles. We begin by giving the definitions of the most important lines in the thermodynamic phase space and the basic equations that describe and govern the interaction between those lines. Details of the derivation of specific expressions are given in the supplementary material. We first consider the stability limit (SL) and the spinodal line. Next, we give the definition of the density anomaly along with the equations that govern its gradient in various projections and note some of the new and important considerations. We then derive the equations which govern the interaction of the stability limit and the spinodal line with the density anomaly. After that we consider the main equations that govern the compressibility anomalies and highlight the expression for the gradient of the compressibility anomaly lines in the $P T$ projection. This naturally leads into the interactions between the density and compressibility anomalies where we derive general equations that govern the interaction between the two types of anomalies. We also recover the three cases in which the compressibility anomaly and density anomaly locus collide from the singularity-free interpretation. ${ }^{4}$ After that the heat capacity anomalies are introduced and expressions for the gradients of the heat capacity anomalies in the $P T$ projection are given. Next, the interaction between the density and heat capacity anomalies are examined. Finally, we consider the interaction between the compressibility and heat capacity anomalies.

\section{A. The stability limit}

The stability limit is the limiting set of thermodynamic conditions at which a metastable phase collapses, effectively defining the boundary for metastable phase existence. In the special case of the mean field, this will coincide with the spinodal line ${ }^{64}$ (see below). The stability limit line does not have any special derivative properties (except when it coincides with the spinodal), and so we can write, in general, an expression for the gradient in the $P T$ projection of any line by expanding the differential of $P$ as a function of $V$ and $T$,

$$
\mathrm{d} P=\left(\frac{\partial P}{\partial T}\right)_{V} \mathrm{~d} T+\left(\frac{\partial P}{\partial V}\right)_{T} \mathrm{~d} V
$$

Now, if we divide by $\mathrm{d} T$ and evaluate at the stability limit (SL), we obtain

$$
\left(\frac{\mathrm{d} P}{\mathrm{~d} T}\right)_{\mathrm{SL}}=\left(\frac{\partial P}{\partial T}\right)_{V}+\left(\frac{\partial P}{\partial V}\right)_{T}\left(\frac{\mathrm{d} V}{\mathrm{~d} T}\right)_{\mathrm{SL}},
$$

which links the gradients of the stability lines in the $P T$ and $V T$ projections. We can also divide by $\mathrm{d} V$ to obtain the $P V$ projection,

$$
\left(\frac{\mathrm{d} P}{\mathrm{~d} V}\right)_{\mathrm{SL}}=\left(\frac{\partial P}{\partial T}\right)_{V}\left(\frac{\mathrm{d} T}{\mathrm{~d} V}\right)_{\mathrm{SL}}+\left(\frac{\partial P}{\partial V}\right)_{T}
$$

We will also be interested in the curvature of the $P T$ and VT projections of the stability limit. Thus, we examine the second order differential of $V$,

$$
\mathrm{d}^{2} V=\left(\frac{\partial^{2} V}{\partial T^{2}}\right)_{P} \mathrm{~d}^{2} T+2 \frac{\partial^{2} V}{\partial T \partial P} \mathrm{~d} T \mathrm{~d} P+\left(\frac{\partial^{2} V}{\partial P^{2}}\right)_{T} \mathrm{~d}^{2} P,
$$

where we used short-hand notation for the mixed second derivative. We divide by $\mathrm{d}^{2} T$ to obtain

$$
\left(\frac{\mathrm{d}^{2} V}{\mathrm{~d} T^{2}}\right)_{\mathrm{SL}}=\left(\frac{\partial^{2} V}{\partial T^{2}}\right)_{P}+2 \frac{\partial^{2} V}{\partial T \partial P}\left(\frac{\mathrm{d} P}{\mathrm{~d} T}\right)_{\mathrm{SL}}+\left(\frac{\partial^{2} V}{\partial P^{2}}\right)_{T}\left(\frac{\mathrm{d}^{2} P}{\mathrm{~d} T^{2}}\right)_{\mathrm{SL}} .
$$

These results will be used to examine the relation between various anomalies and the stability limit.

\section{B. The spinodal line}

The differences between the scenarios used to describe the behavior of the thermodynamic anomalies may often be traced back to the relationship between the anomalies and the spinodal. As a result, although not an anomaly in itself, understanding the interactions between the spinodal line and the density anomaly lines is very important for probing the complete behavior and magnitude of the anomalous region in thermodynamic phase space.

Mechanical stability requires that the isothermal compressibility must always be finite (with corresponding finite fluctuations) and positive. Where this condition is violated, the system becomes mechanically unstable (the fluctuations become infinite) and collapses toward an alternative phase. A set of such points at different thermodynamic conditions is called the spinodal line and each point on that line satisfies the condition

$$
\left(\frac{\partial P}{\partial V}\right)_{T}=0
$$

The spinodal line coincides with the stability limit in the mean field approximation. At the spinodal line, both the compressibility and heat capacity diverge.

The gradient of the spinodal line in the $P T$ projection is given by (see the supplementary material, Sec. I)

$$
\left(\frac{\mathrm{d} P}{\mathrm{~d} T}\right)_{\text {spinodal }}=\left(\frac{\partial P}{\partial T}\right)_{V} .
$$

The spinodal line is the tangent to the $P T$ projection of isochores and the envelope of isochores then forms the spinodal line. This result was first discovered by Skripov ${ }^{65}$ and is important in governing the behavior of the anomalies in the vicinity of the spinodal and Speedy's SLC. ${ }^{53}$ 
The gradient in the VT projection is given by (see the supplementary material, Sec. I)

$$
\left(\frac{\mathrm{d} V}{\mathrm{~d} T}\right)_{\text {spinodal }}=-\frac{\frac{\partial^{2} P}{\partial V \partial T}}{\left(\frac{\partial^{2} P}{\partial V^{2}}\right)_{T}} .
$$

In the VT projection, the gradient of the spinodal line depends on the spinodal branch we choose (denominator) which will be of constant sign depending on which branch we choose (either the maximum or minimum in the $P V$ projection).

\section{The density anomalies}

The density anomalies are defined by loci of extrema (either maxima or minima) in a density vs temperature $(\rho T)$ map of isobars. As a consequence, we can define two lines-temperatures of maximum density (TMD) and temperature of minimum density (TminD)-by

$$
\left(\frac{\partial \rho}{\partial T}\right)_{P}=0
$$

In practice, the TMD and TminD often meet in a continuous fashion $^{58}$ and the combined line which encompasses both the TMD and TminD is referred to simply as the density anomaly path. For every point (defined with respective thermodynamic variables- $T, P, V$ ) on that path or line, Eq. (9) is satisfied. For a fixed number of particles, we can rewrite the above as (see the supplementary material, Sec. II A)

$$
\left(\frac{\partial V}{\partial T}\right)_{P}=0
$$

for which the TMD is now a minimum and TminD a maximum and which is equivalent to (see the supplementary material, Sec. II A)

$$
\left(\frac{\partial P}{\partial T}\right)_{V}=0
$$

Consider now the differential of $V$ as a function of $P$ and $T$,

$$
\mathrm{d} V=\left(\frac{\partial V}{\partial P}\right)_{T} \mathrm{~d} P+\left(\frac{\partial V}{\partial T}\right)_{P} \mathrm{~d} T
$$

Divide by $\mathrm{d} P$ and evaluate on the density anomaly path, which we will denote $\rho_{\text {extr }}$,

$$
\left(\frac{\mathrm{d} V}{\mathrm{~d} P}\right)_{\rho_{\mathrm{extr}}}=\left(\frac{\partial V}{\partial P}\right)_{T}=-V K_{T}
$$

where $K_{T}$ is the isothermal compressibility. Note that this expression is valid for both the TMD and TminD. From this equation, we determine that a density extrema line is an envelope of the VP projection of isotherms. The gradient of the density anomaly line in the $V P$ projection is always negative (since $K_{T}$ and $V$ are always positive).

The gradient of the density anomalies in the $P T$ projection is given by (see the supplementary material, Sec. II B)

$$
\left(\frac{\mathrm{d} P}{\mathrm{~d} T}\right)_{\rho_{\text {extr }}}=-\frac{\left(\frac{\partial^{2} V}{\partial T^{2}}\right)_{P}}{\frac{\partial^{2} V}{\partial P \partial T}},
$$

and the gradient in the $V T$ projection is (see the supplementary material, Sec. II C)

$$
\left(\frac{\mathrm{d} V}{\mathrm{~d} T}\right)_{\rho_{\text {extr }}}=-\frac{\left(\frac{\partial^{2} P}{\partial T^{2}}\right)_{V}}{\frac{\partial^{2} P}{\partial V \partial T}},
$$

and the link with the $P T$ projection is given by

$$
\left(\frac{\mathrm{d} V}{\mathrm{~d} T}\right)_{\rho_{\text {extr }}}=\left(\frac{\mathrm{d} P}{\mathrm{~d} T}\right)_{\rho_{\text {extr }}}\left(\frac{\partial V}{\partial P}\right)_{T}=-V K_{T}\left(\frac{\mathrm{d} P}{\mathrm{~d} T}\right)_{\rho_{\text {extr }}} .
$$

This result tells us that the gradients of the density anomaly lines in the $P T$ and $V T$ projections are always of the opposite signs due to $V$ and $K_{T}$ being strictly positive.

Finally, the second derivative of the density anomaly locus is given by (see the supplementary material, Sec. II D)

$$
\left(\frac{\mathrm{d}^{2} P}{\mathrm{~d} T^{2}}\right)_{\rho_{\text {extr }}}+\left(\frac{\partial^{2} P}{\partial T^{2}}\right)_{V}=\left(\frac{\partial^{2} P}{\partial V^{2}}\right)_{T}\left(\frac{\mathrm{d}^{2} V}{\mathrm{~d} T^{2}}\right)_{\rho_{\text {extr }}} .
$$

The second derivative of the density anomalies loci is coupled between the $P T$ and $V T$ projections. The sign of the $\left(\frac{\partial^{2} P}{\partial T^{2}}\right)$ term determines if we are at a TMD $\left[\left(\frac{\partial^{2} P}{\partial T^{2}}\right)_{V}>0\right]$ or TminD $\left[\left(\frac{\partial^{2} P}{\partial T^{2}}\right)_{V}<0\right]$ line, whereas the sign of $\left(\frac{\partial^{2} P}{\partial V^{2}}\right)_{T}$ strictly depends in which metastable branch we are (for example, it is positive for the "standard" metastable liquid part of thermodynamic phase space close to the liquid-vapor spinodal).

\section{The interaction between the density anomalies and the stability limit} Eq. (2),

We begin by inserting the density anomaly condition into

$$
\left(\frac{\mathrm{d} P}{\mathrm{~d} T}\right)_{\mathrm{SL}}=\left(\frac{\partial P}{\partial V}\right)_{T}\left(\frac{\mathrm{d} V}{\mathrm{~d} T}\right)_{\mathrm{SL}} .
$$

Notice that if the stability limit coincides with the spinodal line, we can insert the stability limit criterion into the previous equation to obtain

$$
\left(\frac{\mathrm{d} P}{\mathrm{~d} T}\right)_{\text {spinodal }}=0 .
$$

The stability limit line in the PT projection will have zero gradient in the limit as it approaches the spinodal line at the point of interception with a density anomaly. A similar conclusion can be reached if we take the gradient of the spinodal line in the PT projection [Eq. (7)] as the isochore tends to a density anomaly 
point $\left[\left(\frac{\partial P}{\partial T}\right)_{V}=0\right]$. Unfortunately this approach cannot be used to obtain the gradient of the spinodal line in the $V T$ projection. The inversion of Eq. (18) does not formally satisfy the implicit function theorem. The limit of $\left(\frac{\partial V}{\partial P}\right)_{T}$ depends from which side we approach it (it can either be $\pm \infty$ ) and, as a result, such an expression cannot be used to evaluate the behavior of the spinodal line in the VT projection in this approach. For further discussion of the implicit function theorem implications, see the supplementary material, Sec. II A.

We can explore the interaction of the spinodal line with the density anomaly using a different approach. Inserting Eq. (SI-19) into Eq. (8) generates

$$
\left(\frac{\mathrm{d} V}{\mathrm{~d} T}\right)_{\text {spinodal }}=\frac{\left(\frac{\partial^{2} P}{\partial T^{2}}\right)_{V}}{\left(\frac{\mathrm{d} V}{\mathrm{~d} T}\right)_{\rho_{\text {extr }}}\left(\frac{\partial^{2} P}{\partial V^{2}}\right)_{T}} .
$$

We can now determine the sign of the $V T$ projection of the spinodal as it collides with the density anomaly as $\left(\frac{\partial^{2} P}{\partial V^{2}}\right)_{T}>0$ for the liquidvapor spinodal on the liquid side of the spinodal. The zero gradient spinodal projection in the VT plane is intercepted by the density anomaly at infinite gradient. An interception point outside of this condition $\left[\right.$ if $\left.\left(\frac{\partial V}{\partial T}\right)_{\rho_{\text {extr }}} \neq \infty\right]$ will force different density anomalies to intercept the spinodal in the VT projection at different gradients. For example, a positive gradient TMD will intercept the spinodal at a positive gradient, and negative gradient TMD will intercept it at negative spinodal gradient. It is also worthwhile noting that this expression cannot be used to evaluate the collision gradient of the $V T$ projection of the spinodal if the density anomaly gradient is zero. At that point, $\left(\frac{\partial^{2} P}{\partial T^{2}}\right)_{V}=0$ and the denominator is zero rendering the fraction ill-defined.

Consider now the second derivative of the stability limit at the intersection with the density anomaly in the $P T$ projection. In the special case where the stability limit coincides with the spinodal, we can insert $\left(\frac{\mathrm{d} P}{\mathrm{~d} T}\right)_{\mathrm{SL}}=0$ into Eq. (5) to obtain

$$
\left(\frac{\mathrm{d}^{2} V}{\mathrm{~d} T^{2}}\right)_{\text {spinodal }}=\left(\frac{\mathrm{d}^{2} P}{\mathrm{~d} T^{2}}\right)_{\text {spinodal }}\left(\frac{\partial^{2} V}{\partial P^{2}}\right)_{T}+\left(\frac{\partial^{2} V}{\partial T^{2}}\right)_{P} .
$$

If we are assuming that we are studying a metastable liquid near the liquid-vapor stability limit, then $\left(\frac{\partial^{2} V}{\partial P^{2}}\right)_{T}>0$ is strictly positive. The TMD $\left[\left(\frac{\partial^{2} V}{\partial T^{2}}\right)_{P}>0\right]$ intercepts a minimum in the $P T$ projection of the spinodal $\left[\left(\frac{\mathrm{d}^{2} P}{\mathrm{~d} T^{2}}\right)_{\text {spinodal }}>0\right]$, and the VT projection of the spinodal has to have a negative second derivative $\left[\left(\frac{\mathrm{d}^{2} V}{\mathrm{~d} T^{2}}\right)_{\text {spinodal }}>0\right]$. If the TMD line intercepts a maximum in the
$P T$ projection of the spinodal $\left[\left(\frac{\mathrm{d}^{2} P}{\mathrm{~d} T^{2}}\right)_{\text {spinodal }}<0\right]$, then the second derivative of the VT projection of the spinodal line could have either sign based on the magnitude of the two competing terms.

If the TminD line $\left[\left(\frac{\partial^{2} V}{\partial T^{2}}\right)_{P}<0\right]$ intercepts the spinodal line at a maximum in the $P T$ projection $\left[\left(\frac{\mathrm{d}^{2} P}{\mathrm{~d} T^{2}}\right)_{\text {spinodal }}<0\right]$, then the second derivative of the $V T$ projection of the spinodal has to be negative $\left[\left(\frac{\mathrm{d}^{2} V}{\mathrm{~d} T^{2}}\right)_{\text {spinodal }}<0\right]$. In case TminD intercepts a minimum in the PT projection of the spinodal $\left[\left(\frac{\mathrm{d}^{2} P}{\mathrm{~d} T^{2}}\right)_{\text {spinodal }}>0\right]$, the sign of the VT projection of the second derivative of the spinodal line could again be of either sign based on the magnitude of the two competing terms.

\section{E. The origin of the density anomaly based on a thermodynamic analysis of the interaction with the spinodal line}

Consider the gradient of the TMD line as it approaches the spinodal. If that region of the phase space is dominated by the influence of the liquid-vapor spinodal, and there is no liquid-liquid critical point in the vicinity, then $\left(\frac{\partial^{2} P}{\partial V^{2}}\right)_{T}>0$. The gradients of the TMD lines in the $V T$ and $P T$ projections still have to be of opposite sign according to Eq. (16). Consider now the second derivative of either density anomaly line (TMD or TminD) in both projections [Eq. (17)]. For a TMD line which only has negative gradient (as in Speedy's SLC) in the PT projection, the second derivative can be either positive or negative, with a positive second derivative corresponding to low pressures close to the liquid side of the liquid-vapor spinodal and a negative second derivative corresponding to high pressures. Consider the case in which the low pressure part of the TMD is close to the liquid branch of the liquid-vapor spinodal. The second derivative of the VP projection of the TMD line then has to be strictly positive [according to Eq. (17) - see Table I].

Consider now the case in which the TMD avoids collision with the liquid-vapor spinodal. After the TMD line passes through

TABLE I. The possible arrangements of the signs for the four terms in Eq. (17) and the corresponding scenarios for the TMD anomaly.

\begin{tabular}{lcccc}
\hline \hline$\left(\frac{\mathrm{d}^{2} P}{\mathrm{~d} T^{2}}\right)_{\rho_{\text {extr }}}$ & $\left(\frac{\partial^{2} P}{\partial T^{2}}\right)_{V}$ & $\left(\frac{\partial^{2} P}{\partial V^{2}}\right)_{T}$ & $\left(\frac{\mathrm{d}^{2} V}{\mathrm{~d} T^{2}}\right)_{\rho_{\text {extr }}}$ & \\
\hline+ & + & + & + & Scenario \\
+ & + & - & - & SLC \\
- & + & + & - & Non-SLC \\
- & + & - & + & \\
- & + & - & - & \\
- & + & + & + & \\
\hline \hline
\end{tabular}


having an infinite gradient in the $P T$ or $V T$ projections, the TMD line changes gradient and sign of the second derivative. Consider the case when the second derivatives are always of opposite signs (such as for the SW liquids-see Figs. 3 and 4). The second derivatives of the density anomaly locus change signs in both projections at infinite TMD locus gradient. Consider the low pressure (high volume, low density) part of TMD line. In the PT projection, the sign of the second derivative of the TMD line is positive but is negative in the VT projection. The only mechanism by which this can happen for a TMD is if $\left(\frac{\partial^{2} P}{\partial V^{2}}\right)_{T}<0$. However, this means that such a point cannot intercept the liquid-vapor spinodal as, at that point, $\left(\frac{\partial^{2} P}{\partial V^{2}}\right)_{T}>0$. This means that the avoidance of a collision between the TMD and liquid-vapor spinodal lines is necessary if the TMD line passes through infinite gradient and changes gradient sign in the case where the signs of second derivatives of the density anomaly lines are always opposite. Obviously, the origin of the density anomaly in such a case cannot be attributed to the liquid-vapor spinodal and the SLC is invalid in such a case (Table I). Notice that this argument is quite general and is independent of the fact that the TMD and TminD have to meet at the point when the gradient of the density anomaly locus is zero.

If the signs of the second derivative of the density anomaly locus are the same, then the collision of the TMD line with the liquidvapor spinodal is still possible (resulting in an SLC-like scenario, and reported for a patchy colloid model-the $2 \mathrm{~A} 9 \mathrm{~B}$ system ${ }^{64}$ ). Note that analogous arguments are valid to describe the behavior of the TminD locus (see Table II).

\section{F. The compressibility anomalies}

Compressibility anomalies are defined as either a temperature of maximum compressibility (TMC) or temperature of minimum compressibility (TminC), satisfying the condition

$$
\left(\frac{\partial K_{T}}{\partial T}\right)_{P}=0
$$

The above expression can be expanded as in singularity-free scenario $^{4}$ (also given in the supplementary material, Sec. III),

$$
\left(\frac{\partial K_{T}}{\partial T}\right)_{P}=-K_{T} \alpha_{P}-\frac{1}{V} \frac{\partial^{2} V}{\partial P \partial T}
$$

TABLE II. The possible arrangements of the signs for the four terms in Eq. (17) and the corresponding scenarios for the TminD anomaly.

\begin{tabular}{lcccc}
\hline \hline$\left(\frac{\mathrm{d}^{2} P}{\mathrm{~d} T^{2}}\right)_{\rho_{\text {extr }}}$ & $\left(\frac{\partial^{2} P}{\partial T^{2}}\right)_{V}$ & $\left(\frac{\partial^{2} P}{\partial V^{2}}\right)_{T}$ & $\left(\frac{\mathrm{d}^{2} V}{\mathrm{~d}^{2}}\right)_{\rho_{\text {extr }}}$ & Scenario \\
\hline- & - & + & - & \\
- & - & - & + & Non-SLC \\
+ & - & + & - & \\
+ & - & - & + & \\
+ & - & - & - & \\
+ & - & + & + & \\
\hline
\end{tabular}

where $\alpha_{P}$ is the thermal expansion coefficient,

$$
\alpha_{P}=\frac{1}{V}\left(\frac{\partial V}{\partial T}\right)_{P} .
$$

Note that this quantity is zero at the density extrema.

The expression that governs the nature of the compressibility anomaly is the second derivative of the compressibility with respect to temperature evaluated at the conditions corresponding to the compressibility anomaly (see the supplementary material, Sec. III A)

$$
\left(\frac{\partial^{2} K_{T}}{\partial T^{2}}\right)_{P}=-\frac{1}{V} \frac{\partial^{3} V}{\partial T^{2} \partial P}-\frac{K_{T}}{V}\left(\frac{\partial^{2} V}{\partial T^{2}}\right)_{P} .
$$

The gradient of the compressibility anomaly lines is given by (see the supplementary material, Sec. III B)

$$
\left(\frac{\mathrm{d} P}{\mathrm{~d} T}\right)_{K_{T}^{\text {extr }}}=-\frac{\left(\frac{\partial^{2} K_{T}}{\partial T^{2}}\right)_{P}}{\frac{\partial^{2} K_{T}}{\partial T \partial P}} .
$$

At the point at which the TMC becomes the TminC line $\left[\left(\frac{\partial^{2} K_{T}}{\partial T^{2}}\right)_{P}=0\right]$, the gradient of the compressibility anomaly locus has to be zero. The denominator of the above expression can also be expanded (see the supplementary material, Sec. III B),

$$
\frac{\partial^{2} K_{T}}{\partial T \partial P}=-\frac{1}{V} \frac{\partial^{3} V}{\partial T \partial P^{2}}+\frac{\alpha_{P}}{V}\left(\frac{\partial^{2} V}{\partial P^{2}}\right)_{T},
$$

and we can obtain the final expression for the gradient by inserting Eqs. (27) and (25) into the expression for the gradient of the compressibility anomaly,

$$
\left(\frac{\mathrm{d} P}{\mathrm{~d} T}\right)_{K_{T}^{\text {extr }}}=\frac{\frac{\partial^{3} V}{\partial T^{2} \partial P}+K_{T}\left(\frac{\partial^{2} V}{\partial T^{2}}\right)_{P}}{\frac{\partial^{3} V}{\partial T \partial P^{2}}-\alpha_{P}\left(\frac{\partial^{2} V}{\partial P^{2}}\right)_{T}} .
$$

\section{G. The heat capacity anomalies}

The heat capacity anomaly is defined as the locus of the isobaric heat capacity extrema along isotherms (TMH-temperature of maximum heat capacity and $\mathrm{TminH}$ - temperature of minimum heat capacity),

$$
\left(\frac{\partial C_{P}}{\partial P}\right)_{T}=T \frac{\partial^{2} S}{\partial P \partial T}=0,
$$

where we can rewrite the above condition to read (see the supplementary material, Sec. IV A)

$$
\left(\frac{\partial C_{P}}{\partial P}\right)_{T}=-T\left(\frac{\partial^{2} V}{\partial T^{2}}\right)_{P}
$$

The second derivative of the heat capacity informs us as to whether we are at a TMH or TminH point (see the supplementary material, Sec. IV A), 


$$
\left(\frac{\partial^{2} C_{P}}{\partial P^{2}}\right)_{T}=T \frac{\partial^{3} S}{\partial P^{2} \partial T}=-T \frac{\partial^{3} V}{\partial T^{2} \partial P} .
$$

To obtain the gradient of the heat capacity anomaly, we apply a similar procedure as in the case of the compressibility anomaly to obtain (see the supplementary material, Sec. IV B)

$$
\left(\frac{\mathrm{d} P}{\mathrm{~d} T}\right)_{C_{P}^{\text {extr }}}=-\frac{\frac{\partial^{2} C_{P}}{\partial T \partial P}}{\left(\frac{\partial^{2} C_{P}}{\partial P^{2}}\right)_{T}}
$$

From this, it is clear that at the crossover point at which the TMH becomes the $\operatorname{TminH}\left[\left(\frac{\partial^{2} C_{P}}{\partial P^{2}}\right)_{T}=0\right]$, the gradient of the heat capacity anomaly locus in the $P T$ projection has to be infinite. In order to obtain the full expression, we have to expand the numerator (see the supplementary material, Sec. IV B),

$$
\left(\frac{\mathrm{d} P}{\mathrm{~d} T}\right)_{C_{P}^{\text {extr }}}=T \frac{\left(\frac{\partial^{3} V}{\partial T^{3}}\right)_{P}}{\left(\frac{\partial^{2} C_{P}}{\partial P^{2}}\right)_{T}}=-\frac{\left(\frac{\partial^{3} V}{\partial T^{3}}\right)_{P}}{\frac{\partial^{3} V}{\partial T^{2} \partial P}} .
$$

\section{$H$. The interactions between the density and the compressibility anomalies}

To consider the interaction between the density and the compressibility anomalies, we begin by combining Eqs. (14) and (23) to obtain an equation which was first reported for the singularity-free scenario,

$$
\left(\frac{\partial K_{T}}{\partial T}\right)_{P}=\frac{1}{V} \frac{\left(\frac{\partial^{2} V}{\partial T^{2}}\right)_{P}}{\left(\frac{\mathrm{d} P}{\mathrm{~d} T}\right)_{\rho_{\mathrm{ext}}}} .
$$

The implications of this equation are following. If the density and compressibility anomalies are to collide, then both sides have to be zero. In order for the right-hand side to be zero, $\left(\frac{\mathrm{d} P}{\mathrm{~d} T}\right)_{\rho_{\text {extr }}}$ has to be infinite. If the numerator on the right-hand side is zero, then the denominator is also zero according to Eq. (14), which produces an undefined quantity. As a result, the compressibility anomaly collides with the density anomaly where the latter has infinite gradient.

If we evaluate the expression for the gradient of the compressibility anomaly locus [Eqs. (26) and (28)] at the density anomaly $\left(\alpha_{P}=0\right)$, we obtain

$$
\left(\frac{\mathrm{d} P}{\mathrm{~d} T}\right)_{K_{T}^{\operatorname{expr}}}=\frac{\left(\frac{\partial^{2} K_{T}}{\partial T^{2}}\right)_{P}}{\frac{1}{V} \frac{\partial^{3} V}{\partial T \partial P^{2}}}=\frac{\frac{\partial^{3} V}{\partial T^{2} \partial P}+K_{T}\left(\frac{\partial^{2} V}{\partial T^{2}}\right)_{P}}{\frac{\partial^{3} V}{\partial T \partial P^{2}}} .
$$

Consider the value of $\frac{\partial^{3} V}{\partial T \partial P^{2}}$ at an infinite gradient density anomaly line in either the $P T$ or $V T$ projections. If such a point is a maximum TMD temperature or minimum TminD temperature (see the two intersection points of the density anomaly lines-red with the vertical blue lines in Fig. 2), then $\frac{\partial^{3} V}{\partial T \partial P^{2}}>0$ (as can be concluded from the inset of Fig. 2). If the TMD collides with a compressibility locus of zero gradient, this will correspond to the inflection point (neither a TMC nor TminC). Whereas if the TMD collides with a positive gradient compressibility locus, that point has to then correspond to a TminC point $\left[\left(\frac{\partial^{2} K_{T}}{\partial T^{2}}\right)_{P}>0\right]$, and if the TMD collides with a negative gradient compressibility locus, that point must correspond to a TMC point $\left[\left(\frac{\partial^{2} K_{T}}{\partial T^{2}}\right)_{P}<0\right]$. This recovers three collision cases reported in singularity-free interpretation. ${ }^{4}$ As we can see, the recovery of the behavior of the compressibility and density loci collision is conditional on the shape of the density anomaly locus but is agnostic to the density anomaly considered (be it TMD or TminD). Notice that if the TMD and TminD are to change places, the sign of the $\frac{\partial^{3} V}{\partial T \partial P^{2}}$ term would then be negative. This would in turn change the signs of the gradients at which TMC and TminC collide with the density anomaly. In the case of a SLClike monotonic density anomaly locus that has an infinite gradient but does not retract, the sign of $\frac{\partial^{3} V}{\partial T \partial P^{2}}$ is not obvious.

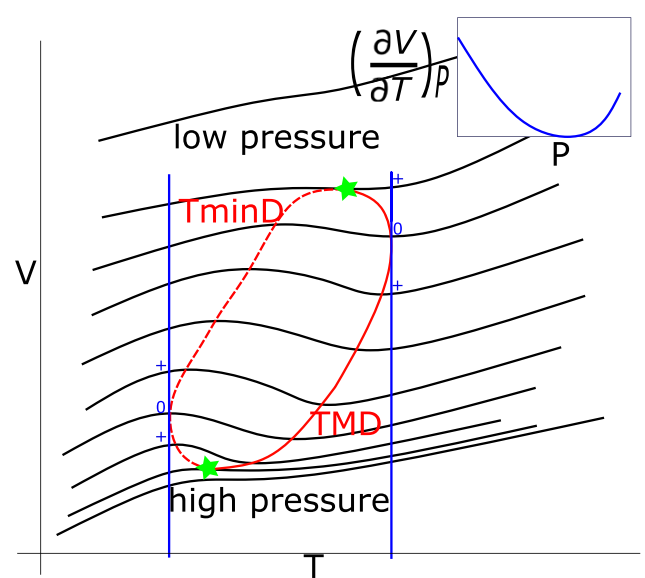

FIG. 2. Schematic diagram of the VT projection of the density anomaly loci which avoids collision with the liquid-vapor spinodal. The black lines represent the isobars, whilst the red lines represent both the TMD (full) and TminD (dashed) loci and which correspond to extrema on the isobars. The green stars highlight the points at which the gradient of the density anomaly locus is zero, at which poin the TMD changes to TminD and where they collide with the heat capacity anomaly locus. The vertical blue lines highlight the minimum and maximum temperatures at which a density anomaly is present and coincides with the infinite gradient for the density anomaly loci (and at which points the compressibility anomaly loci collide). The blue signs (+ and 0) next to the blue vertical lines denote the sign of the partial derivative of volume with respect to temperature at constant pressure, at the points where vertical blue line intersects the isobars. The inset shows this derivative as a function of pressure at constant temperature deducted from the sign analysis. 


\section{The interactions between the density and the heat capacity anomalies}

To consider the interaction between the density and the heat capacity anomalies, we begin by applying the heat capacity anomaly condition $\left(\frac{\partial C_{P}}{\partial P}\right)_{T}=-T\left(\frac{\partial^{2} V}{\partial T^{2}}\right)_{P}=0$ to the gradient of the density anomaly in the $P T$ projection [Eq. (14)],

$$
\left(\frac{\mathrm{d} P}{\mathrm{~d} T}\right)_{\rho_{\text {extr }}}=-\frac{\left(\frac{\partial^{2} V}{\partial T^{2}}\right)_{P}}{\frac{\partial^{2} V}{\partial P \partial T}}=0 .
$$

The heat capacity anomaly strictly intersects the density anomaly at zero gradient in the $P T$ projection.

Consider again the gradient of the heat capacity anomaly [Eq. (32)],

$$
\left(\frac{\mathrm{d} P}{\mathrm{~d} T}\right)_{C_{P}^{\text {extr }}}=-\frac{\frac{\partial^{2} C_{P}}{\partial T \partial P}}{\left(\frac{\partial^{2} C_{P}}{\partial P^{2}}\right)_{T}}=\frac{T\left(\frac{\partial^{3} V}{\partial T^{3}}\right)_{P}}{\left(\frac{\partial^{2} C_{P}}{\partial P^{2}}\right)_{T}} .
$$

Consider the isobars in the VT projection at the point at which the gradient of the density anomaly is zero (marked by green stars in Fig. 2) and intersects the heat capacity anomaly. The isobar has an inflection at the point of zero density anomaly gradient where the TMD and TminD meet. ${ }^{58}$ By definition, such a point needs to have the lowest nonzero derivative to be of odd order (in our case third order). In our case, the sign of the third derivative at the inflection point of the isobar is positive (the first derivative is positive to the both left and right of that point as expected for a liquid outside anomalous regions as can be seen in Fig. 2). As a result, we conclude that

$$
\frac{\partial^{2} C_{P}}{\partial T \partial P}=-T\left(\frac{\partial^{3} V}{\partial T^{3}}\right)_{P}<0 .
$$

This enables us to recover three cases in which the heat capacity anomaly locus intercepts the density anomaly locus, noted previously but not explicitly derived. ${ }^{60}$ If, at the interception point, $\left(\frac{\partial^{2} C_{P}}{\partial P^{2}}\right)_{T}=0$ (corresponding to the transition between TMH and TminH), the gradient of the heat capacity locus has to be infinite. A $\mathrm{TMH}$ line that intersects the density anomaly locus needs to have a negative gradient in the $P T$ projection, while the TminH line needs to have a positive gradient in the $P T$ projection.

In the analogue of the manner in which compressibility anomaly loci collide with the density anomaly loci, the dependence of the gradient of the heat capacity anomaly loci on collision with the density anomaly loci is conditional on the shape of the density anomaly locus. If again the TMD and TminD are to swap places, the gradients of the heat capacity anomalies would have to change signs when colliding with the density anomaly locus.

\section{J. The origin of compressibility and heat capacity anomalies}

Compared to the density anomaly, both the TminC and $\mathrm{TminH}$ loci could have a much simpler origin. Considering the definition of both lines, then the TminC is a minimum compressibility at constant pressure, whereas the $\mathrm{TminH}$ is a minimum heat capacity at constant temperature. At a spinodal, both the heat capacity and compressibility will diverge. As a result, nonmonotonic or multiple spinodal lines can generate TminC and TminH points. To put this in the perspective of a $P T$ projection, one has to consider the location of the spinodal lines. If a part of thermodynamic space contains two spinodal points (not necessarily belonging to the same spinodal line) at the same pressure and two different temperatures, a TminC point at a temperature in between the two temperatures has to be present. An analogous argument can be constructed to identify a TminH point in between two spinodal points at the same temperature and at two different pressures.

A tensile instability must necessarily generate TminC points. In the SLC scenario, the TminC locus should terminate at the spinodal minimum (although the TminC is not strictly present at the spinodal itself) in the $P T$ projection (at the point where the TMD terminates, the TMD being present at the spinodal). As a result, we should not treat such a point as a collision between the respective density and compressibility anomalies. Similarly the high pressure TMD termination in the SLC scenario (the "kink") also necessarily generates TminH points.

On the other hand, TMC and TMH lines are often associated with the extension of the spinodal lines beyond the critical point in which the first order transition and the spinodal lines terminate. If one considers the case in the PT projection in which we have two spinodal points at the same pressure at low and high temperatures, and we have a TMC point at a temperature in between, there must exist two TminC points at temperatures between the lower spinodal and the TMC temperature as well as one between the TMC and high spinodal temperatures. As a result, the TMC line would be surrounded by TminC lines. The analogous principle applies to the $\mathrm{TMH}$ points considered at constant temperature.

Finally, one must consider the role of the density anomaly. Looking at Eq. (14), we can clearly see that density anomaly with zero gradient requires the existence of a heat capacity anomaly point. Similarly, an infinite gradient density anomaly requires the existence of the compressibility anomaly at that point [Eq. (34)]. As a result, while compressibility and heat capacity anomalies can exist without the density anomaly, certain density anomaly scenarios can guarantee that they will be present.

\section{K. The interaction between the heat capacity and the compressibility anomalies}

We begin by inserting the condition for the heat capacity anomaly $\left[\left(\frac{\partial C_{P}}{\partial P}\right)_{T}=-T\left(\frac{\partial^{2} V}{\partial T^{2}}\right)_{P}=0\right]$ into the second derivative of the compressibility evaluated at the compressibility anomaly [Eq. (25)],

$$
\left(\frac{\partial^{2} K_{T}}{\partial T^{2}}\right)_{P}=-\frac{1}{V} \frac{\partial^{3} V}{\partial T^{2} \partial P} .
$$


If we insert this into the equation for the second derivative of the heat capacity with respect to the pressure, which tells us which heat capacity anomaly we are considering [Eq. (31)], we obtain

$$
\left(\frac{\partial^{2} C_{P}}{\partial P^{2}}\right)_{T}=T V\left(\frac{\partial^{2} K_{T}}{\partial T^{2}}\right)_{P}
$$

Given that both $V$ and $T$ must be strictly positive, this means that the intersection between compressibility and heat capacity anomalies can only happen between lines of minimum compressibility and minimum heat capacity or between the lines of maximum compressibility or maximum heat capacity. Notice that we do not require that $K_{T}$ or $C_{P}$ go to infinity if the compressibility maximum and heat capacity maximum collide, which means that the TMC and TMH can collide away from the critical point.

Consider now the gradient for the compressibility anomaly [Eq. (26)]. If we now substitute Eq. (40) into Eq. (26), we get

$$
\left(\frac{\mathrm{d} P}{\mathrm{~d} T}\right)_{K_{T}^{\text {extr }}}=-\frac{\left(\frac{\partial^{2} C_{P}}{\partial P^{2}}\right)_{T}}{T V \frac{\partial^{2} K_{T}}{\partial T \partial P}} .
$$

Inverting Eq. (32) and inserting it into the above equation gives

$$
\left(\frac{\mathrm{d} P}{\mathrm{~d} T}\right)_{K_{T}^{\text {extr }}}=\frac{\frac{\partial^{2} C_{P}}{\partial T \partial P}}{T V \frac{\partial^{2} K_{T}}{\partial T \partial P}\left(\frac{\mathrm{d} P}{\mathrm{~d} T}\right)_{C_{P}^{\text {extr }}}} .
$$

Finally, we can substitute Eq. (SI-48) and Eq. (27), onto which we also apply the heat capacity anomaly condition, into the above equation to obtain

$$
\left(\frac{\mathrm{d} P}{\mathrm{~d} T}\right)_{K_{T}^{\text {extr }}}=-\frac{\left(\frac{\partial^{3} V}{\partial T^{3}}\right)_{P}}{\left(-\frac{\partial^{3} V}{\partial T \partial P^{2}}+\alpha_{P}\left(\frac{\partial^{2} V}{\partial P^{2}}\right)_{T}\right)\left(\frac{\mathrm{d} P}{\mathrm{~d} T}\right)_{C_{P}^{\text {extr }}}} .
$$

The first conclusion is that for a zero gradient heat capacity anomaly line, the point of intersection for the compressibility anomaly needs to have an infinite gradient in the $P T$ projection and vice versa. Outside of the special cases for zero and infinite gradient compressibility and heat capacity anomaly loci, the signs of the said anomalous lines at their collision point will depend on the signs of $\frac{\partial^{2} C_{P}}{\partial T \partial P}$ and $\frac{\partial^{2} K_{T}}{\partial T \partial P}$ although the signs of these two terms are nontrivial to predict except for some specific points associated with the density anomaly (such as when it has an infinite or zero gradient). As a result, prediction of the signs of the gradients of the compressibility and heat capacity anomalies on collision is difficult.

Finally, we consider the case in which the compressibility anomaly locus collides with the heat capacity anomaly locus at a point where TminC and TMC meet. At such a point, the TMH and TminH also merge [Eq. (40)] and so

$$
\left(\frac{\partial^{2} C_{P}}{\partial P^{2}}\right)_{T}=\left(\frac{\partial^{2} K_{T}}{\partial T^{2}}\right)_{P}=0
$$

Recall now that when $\left(\frac{\partial^{2} K_{T}}{\partial T^{2}}\right)_{P}=0$, the gradient of compressibility anomaly locus needs to be zero in the PT projection [Eq. (26)]. Similarly when $\left(\frac{\partial^{2} C_{P}}{\partial P^{2}}\right)_{T}=0$, the heat capacity anomaly needs to have an infinite gradient in the $P T$ projection [Eq. (32)]. Thus, if the compressibility and the heat capacity anomaly collide at a point where both minima change to maxima, they will be orthogonal to each other in the $P T$ projection.

\section{NUMERICAL RESULTS}

Having established mathematically a range of conditions under which the various anomalies interact, we will now demonstrate the validity of these results by performing a range of numerical simulations using a commonly employed potential model.

\section{A. Potential models and methods}

We employ a Stillinger-Weber (SW) potential $^{36}$ which may be most simply written as the sum of 2 - and 3-body interactions,

$$
\begin{aligned}
U & =\sum_{i, j>i} U_{2}\left(r_{i j}\right)+\lambda \sum_{i, j \neq i, k>j} U_{3}\left(r_{i j}, r_{i k}, \theta_{i j k}\right), \\
U_{2}\left(r_{i j}\right) & =A\left[B\left(\frac{1}{r_{i j}}\right)^{p}-\left(\frac{1}{r_{i j}}\right)^{q}\right] \mathrm{e}^{\frac{1}{r_{i j}-a}}, \\
U_{3}\left(r_{i j}, r_{i k}, \theta_{i j k}\right) & =\left[\cos \theta_{i j k}-\cos \theta_{0}\right]^{2} \mathrm{e}^{\frac{\gamma}{r_{i j}-a}} \frac{\gamma}{\mathrm{e}^{r_{i k}-a}} .
\end{aligned}
$$

Here, the SW parameters are fixed at those obtained originally to model silicon ${ }^{36}$ with the exception of the strength of the three-body term $(\lambda)$. The values of $\lambda$ investigated in this work are $\lambda=18$ and 26 (compared to the value for silicon $\lambda=21$ ) and are chosen to reflect the approximate lower and upper ranges of the parameter $\lambda$ for which anomalous thermodynamic properties are observed. ${ }^{30}$ At these values of $\lambda$, we observe some novel interactions between certain thermodynamic anomalies which make these two parameterizations perfect for testing our theoretical predictions. We use molecular dynamics (MD) in the NVT ensemble to generate the required thermodynamic data using LAMMPS $^{66}$ with the temperature maintained using Nosé-Hoover thermostats. ${ }^{67,68}$ In order to calculate isochores, the volume and number of atoms $(N=512)$ is kept constant, while the temperature is varied systematically. A range of densities (volumes) are investigated in order to obtain a full set of isochores within the stability limit of the potential models. The initial simulation cell is created from a diamond crystal unit cell copied $4 \times 4 \times 4$ times in the respective dimensions. The temperature is changed in increments of 0.002 reduced units, and the volume is changed by increasing the length of the unit cell of the diamond crystal in increments of 0.00477 reduced units. An integration time step of $0.383 \mathrm{fs}$ is applied throughout. The system is equilibrated using simulations of $t \sim 2$ ns with production runs of $t \sim 2.5$ ns. State points for which dynamical arrest was observed on the simulation time scale were also investigated using replica exchange molecular dynamics (REMD), ${ }^{62,63}$ coupling to higher temperature states to potentially enable a more complete exploration 
of the liquid state configurational space. The original REMD simulations were $t \sim 4 \mathrm{~ns}$ long, and the end of "standard" MD simulations was used as a starting point. We have performed very long REMD simulations of $t \sim 40 \mathrm{~ns}$ in which spontaneous crystallization is observed. It should be noted that estimating the quality of the sampling on the basis of diffusion can be problematic due to any partial crystallization of the system. To improve our sampling, we perform eight REMD simulations of $\sim 4 \mathrm{~ns}$ each for each thermodynamic state point and average over these. In each case, a different starting configuration was extracted from the "standard" MD runs. Such sampling is always preferred to just increasing the length of the simulation, ${ }^{69}$ but this is also a useful strategy to avoid crystallization while increasing the sampling time.

When determining the locations of the respective anomaly loci and the stability limits, we use the thermodynamic data from long REMD simulations (if available) and switch to data from "standard" MD simulations for higher temperatures. The choice of the temperatures at which REMD and MD data converge is selected such that the system diffuses at this temperature across the whole volume range considered. Points on an isochore are filtered using a second order Butterworth filter and a 4th order univariate spline fitted to the filtered data. The first derivative is then calculated and identified as a maxima or minima from the sign of the second derivative. These correspond to points on the TMD or TminD lines. A similar procedure is employed to locate the compressibility anomalies (TMC and TminC). Isotherms are interpolated using a 4th order spline fit and the derivative determined in order to obtain the isothermal compressibility. We then recast the obtained compressibility data on a pressure and temperature grid and identify extrema of compressibility with respect to temperature at constant pressure using the analogous procedure to that used to identify the density anomalies. For numerical ease, an analogous procedure was employed to consider the heat capacity anomalies, the heat capacity being obtained using the expression

$$
C_{P}=\left(\frac{\partial U}{\partial T}\right)_{V}+T\left(\frac{\partial P}{\partial T}\right)_{V}\left(\frac{\partial V}{\partial T}\right)_{P}
$$

The liquid-vapor stability limits were calculated by determining the lowest pressure point on an isotherm for each temperature. We note that this approach gives only an upper bound estimate of the liquidvapor-spinodal line in the $P T$ plane.

\section{B. Results}

In this section, we report the main features of numerically studied systems in terms of lines of anomalous behavior and limits of stability. Figures 3 and 4 show the anomaly loci and stability limits for $\lambda=26$ and $\lambda=18$ in both the (a) $P T$ and (b) VT projections. Let us consider the interactions of the anomalies with each other and with the stability limits.

The limit of stability at high temperatures for $\lambda=26$ seems to be very close to the true spinodal line. However, as the temperature is lowered and we start approaching the anomalous region, the simulations start to cavitate (below $T^{*}=0.11$, corresponding to $T<2750 \mathrm{~K}$ for $\lambda=26$ ). For $\lambda=18$, cavitation is observed at all temperatures

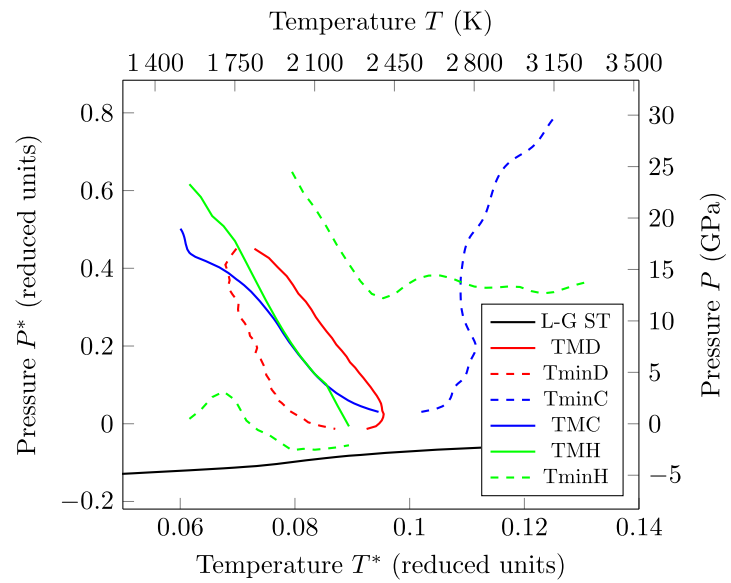

(a)

Temperature $T(\mathrm{~K})$

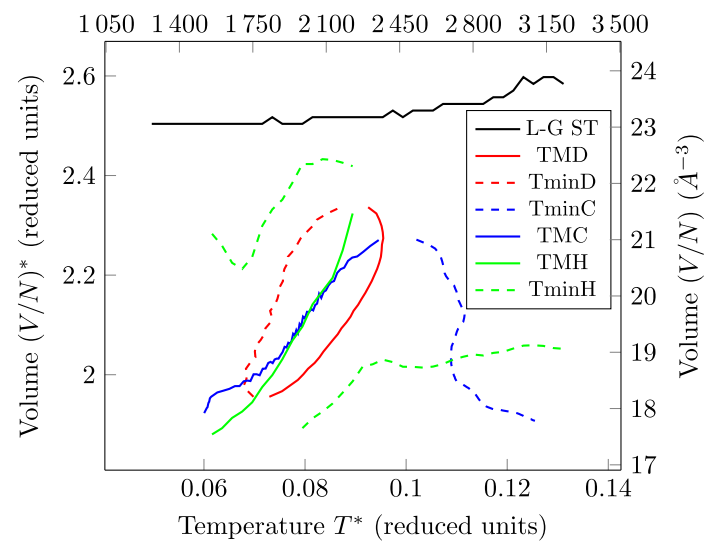

(b)

FIG. 3. Thermodynamic anomaly loci for the modified Stillinger-Weber potentia with $\lambda=26$ shown in the (a) $P T$ and (b) VT projections. The black lines highlight the liquid-vapor stability limit. The minimum and maximum loci for a given anomaly are displayed as dashed and solid lines, respectively. Key: red-density anomalies, blue-compressibility anomalies, and green-heat capacity anomalies.

at low density (high volume). In these cases, the effective interaction between the density anomalies and the stability limit is actually the interaction between the density anomalies and the cavitation line.

For $\lambda=26$, the collision between the density anomaly and the stability limit is avoided and the liquid-vapor stability limit is monotonically decreasing in the $P T$ and $V T$ projections of the phase diagram. For $\lambda=18$, the stability limit (or cavitation line) retracts giving a tensile instability-like behavior characteristic of that predicted by the SLC. However, the tensile instability terminates in a maximum and starts monotonically decreasing thereafter, unlike in the SLC case. It is unclear if the true spinodal for this system behaves in the same way as the observed cavitation line. However, analyzing the signs of the second derivatives of the high pressure TminD line (see Sec. II E) suggests that the origin of the density anomaly cannot be 


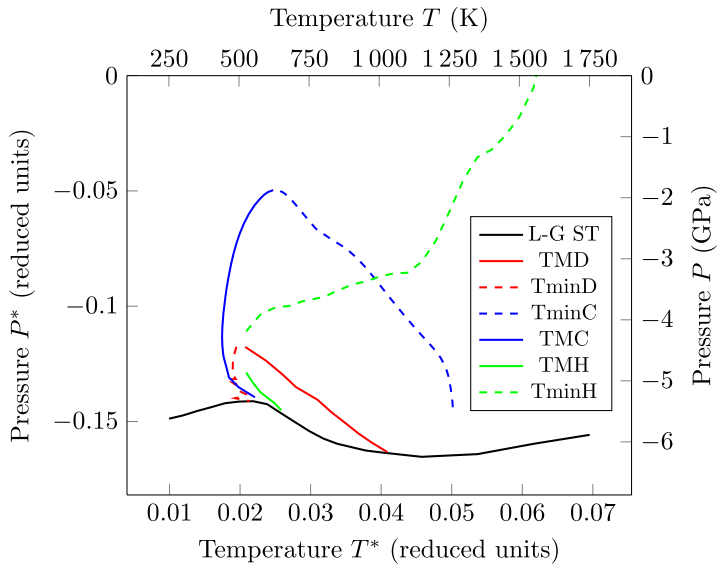

(a)

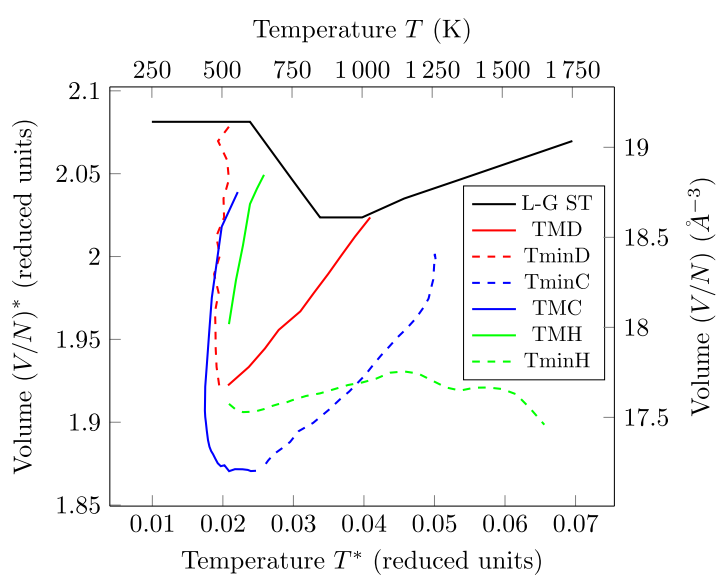

(b)

FIG. 4. Thermodynamic anomaly loci for the modified Stillinger-Weber potential with $\lambda=18$ shown in the (a) $P T$ and (b) VT projections. The black lines highlight the liquid-vapor stability limit. The minimum and maximum loci for a given anomaly are displayed as dashed and solid lines, respectively. Key: red-density anomalies, blue-compressibility anomalies, and green-heat capacity anomalies.

the liquid-vapor spinodal as the TminD meets with the TMD line at a maximum (negative second derivative) in the $P T$ projection and at minimum in the $V T$ projection (positive second derivative). This would suggest that the true spinodal of the system actually avoids the collision with the density anomaly locus, possibly giving us a similar scenario for the density anomalies as observed for $\lambda=26$ case.

At $\lambda=18$, the density anomaly lines intersect the stability limit (or cavitation line in this case) when the latter has zero gradient in the PT plane (see Sec. II D). The TMD line collides at stability limit minimum, whilst the TminD line collides at the stability limit maximum in the $P T$ projection. The respective temperatures for these intersections for the TMD and TminD lines are $\left\{p^{*}, T^{*}\right\} \sim\{-0.14$, $0.041\}$ and $\left\{p^{*}, T^{*}\right\} \sim\{-0.165,0.022\}$ (corresponding to $\{p, T\}$ $\sim\{-5.3 \mathrm{GPa}, 1030 \mathrm{~K}\}$ and $\{p, T\} \sim\{-6.2 \mathrm{GPa}, 550 \mathrm{~K}\}$, respectively). In the $V T$ projection, the positive gradient TMD line intersects the stability limit at positive gradient, while a positive gradient
TminD line intersects the stability limit at negative gradient (due to low resolution of our $V$ grid, this can not be clearly seen) just as we predicted from purely theoretical considerations earlier.

It is quite remarkable that the cavitation line follows similar rules to the spinodal line when colliding with the density anomaly. It should be noted that the estimated "true" spinodal would lie at lower pressures than the reported stability limit or the cavitation line for both values of $\lambda$. At $\lambda=26$, the density anomaly avoids collision with the observed limit of stability.

Besides collision with the spinodal line (stability limit), the only other mechanism by which the TMD or TminD loci can terminate is to merge. ${ }^{58}$ For both values of $\lambda$, the TMD and TminD meet at high pressures in the $P T$ projection $(\{p, T\} \sim\{-4.1 \mathrm{GPa}$, $500 \mathrm{~K}\}$ corresponding to $\left\{p^{*}, T^{*}\right\} \sim\{-0.12,0.02\}$ for $\lambda=18$ and $\{p, T\} \sim\{18 \mathrm{GPa}, 1750 \mathrm{~K}\}$ corresponding to $\left\{p^{*}, T^{*}\right\} \sim\{0.47$, $0.07\}$ for $\lambda=26)$ and low volumes in the $V T$ projection $(\{V, T\}$ $\sim\left\{18.5 \AA^{3}, 500 \mathrm{~K}\right\}$ corresponding to $\left\{V^{*}, T^{*}\right\} \sim\{1.925,0.02\}$ for $\lambda=18$, and $\{V, T\} \sim\left\{18 \AA^{3}, 1750 \mathrm{~K}\right\}$ corresponding to $\left\{V^{*}, T^{*}\right\}$ $\sim\{1.94,0.07\}$ for $\lambda=26) . \lambda=26$ also offers a more widely studied meeting of the TMD and TminD loci at low pressures and high volume $\left(\{p, V, T\} \sim\left\{0 \mathrm{GPa}, 21.9 \AA^{3}, 2250 \mathrm{~K}\right\}\right.$ corresponding to $\left.\left\{p^{*}, V^{*}, T^{*}\right\} \sim\{0,2.32,0.09\}\right)$ as the collision with the stability limit is avoided in the manner of the second LLCP scenario. By employing an analysis of the second derivatives to find the origin of the density anomaly, we can clearly see that $\lambda=26$ belongs in the nonSLC scenario. The minima in the density anomaly loci in the $P T$ projection correspond to a maxima in the $V T$ projection, and we identify the non-SLC cases for both extrema. It should be noted, however, that we detect no traces of a liquid-liquid critical point. As a result, the density anomaly locus for $\lambda=26$ creates a continuous loop.

The two compressibility anomaly lines meet above the density anomaly locus and both terminate at the stability limit in the $P T$ projection for $\lambda=18$. In standard second liquid-liquid critical point scenario or singularity-free interpretation, the compressibility anomalies meet only once close to the infinite TMD gradient instead of meeting above the density anomaly locus.

At $\lambda=18$ (Fig. 4), the intersection of the TMD and TminC loci is obscured by the stability limit. However, the intersection of the TminD and TMC loci (something generally not observed for nonSLC scenarios) is seen at $\left\{p^{*}, T^{*}\right\} \sim\{-0.13,0.02\}$ (corresponding to $\{p, T\} \sim\{-4.9 \mathrm{GPa}, 500 \mathrm{~K}\}$ ) with TMC having negative gradient in the $P T$ projection as predicted. The low pressure TminC line seems to terminate abruptly. The reason for this is our inability to effectively construct splines in the region of the thermodynamic phase space where the stability limit is retracting.

The crossover from the TMC to TminC for $\lambda=26$ appears at a higher temperature than the maximum temperature TMD point (corresponding to TEC case III in Ref. 4). As a result, the TMC locus has negative gradient (in the $P T$ projection) when colliding with the density anomaly as Ref. 4 and ourselves predict.

The density and compressibility anomalies collide when the gradient of the respective TminD and TMD lines are infinite (see Sec. II H). These intersections are clearest at $\lambda=26$ (Fig. 3) with respective pressures and temperatures for the crossing of the compressibility anomalies with the TMD and TminD lines of $\left\{p^{*}, T^{*}\right\}$ $\sim\{0.02,0.095\}$ and $\left\{p^{*}, T^{*}\right\} \sim\{0.40,0.070\}$ (corresponding to $\{p, T\}$ $\sim\{0.8 \mathrm{GPa}, 2390 \mathrm{~K}\}$ and $\{p, T\} \sim\{15 \mathrm{GPa}, 1760 \mathrm{~K}\}$, respectively). The 
TMC line has a negative gradient throughout the thermodynamic space studied in the PT projection. The TminC line starts out with positive gradient at low pressure, briefly retracts with negative gradient crossing the TMC locus, and then continues to positive gradient at high pressures.

The crossover between the TMH and TminH occurs at infinite gradient in both the $P T$ and $V T$ projections (see Sec. II G). For $\lambda=26$, only the low pressure crossover is observed at $\left\{p^{*}, T^{*}\right\}$ $\sim\{-0.03,0.09\}$ (corresponding to $\{p, T\} \sim\{-1.2 \mathrm{GPa}, 2240 \mathrm{~K}\}$ ). Any high pressure crossover occurs in a region of the phase space for which the heat capacity anomalies become too weak to effectively observe. The TMH points at constant temperature slices are "surrounded" by TminH lines as argued previously (see Sec. II J) for both values of $\lambda$. For $\lambda=18$, any low pressure crossover is obscured by the stability limit. The high pressure crossover is observed at $\left\{p^{*}, T^{*}\right\}$ $\sim\{-0.05,0.025\}$ (corresponding to $\{p, T\} \sim\{-1.9 \mathrm{GPa}, 630 \mathrm{~K}\}$ ).

The density and heat capacity anomalies collide when the gradient of the density anomaly line is zero at points where the TMD and TminD loci meet (see Sec. II I). For $\lambda=26$, both the upper and lower intersections are observed at $\left\{p^{*}, T^{*}\right\} \sim\{0,0.090\}$ and $\left\{p^{*}, T^{*}\right\} \sim\{0.45,0.074\}$ (corresponding to $\{p, T\} \sim\{0 \mathrm{GPa}, 2260 \mathrm{~K}\}$ and $\{p, T\} \sim\{17 \mathrm{GPa}, 1860 \mathrm{~K}\}$, respectively). In both cases, the $\mathrm{TMH}$ line has a negative gradient when colliding with the density anomaly locus (as predicted in Sec. II I). For $\lambda=18$, only the high pressure interaction is observed at $\left\{p^{*}, T^{*}\right\} \sim\{-0.118,0.020\}$ (corresponding to $\{p, T\} \sim\{-4.4 \mathrm{GPa}, 500 \mathrm{~K}\}$ ), the low pressure interaction being obscured by the intersection with the stability limit. The gradient of the heat capacity locus is infinite when colliding with the density anomaly locus, again in accordance with earlier predictions.

We observe the predicted collisions between the TminH and TminC for both $\lambda$ values and TMC and TMH for $\lambda=26$ (see Sec. II K). For $\lambda=26$, the two loci cross with a TminH gradient of approximately zero and a TminC gradient of around infinity, again as predicted. For $\lambda=18$, the crossover is more complex although the respective gradients appear near orthogonal. We also observe the collision of TMC and TMH lines enclosed by the density anomaly region. In the second LLCP scenario, this would be expected to generate a LLCP; however, we do not observe such behavior.

\section{CLASSIFICATION SCHEME FOR ANOMALIES}

Finally, let us consider a more general picture of the anomalies studied here. The compressibility and heat capacity anomalies are defined in a similar manner (as cross second derivatives in terms of pressure and temperature) and so interact with the density anomalies in an analogous manner, for example, intercepting the density anomaly at zero or infinite gradient. Furthermore, both the compressibility and heat capacity anomalies can exist independently of any density anomalies. The density anomalies may form complete loops or collide with the stability limit depending upon the balance of the two- and three-body interactions. As a result, the density anomalies and the respective compressibility and heat capacity anomalies behave in a fundamentally different manner and so may be classified as first and second order anomalies, respectively, according to the order of the highest derivative of the basic thermodynamic quantity with respect to which they are defined. As a result, the definitions for the density anomalies [Eqs. (9) and (10) or Eq. (11)] define this as the first order. On the other hand, once we expand the full expression for the compressibility anomalies [Eq. (23)], we see that the highest order derivative is the second order. Finally, according to Eq. (30), an analogous argument is valid for the heat capacity anomalies. In addition, we note that both the compressibility and heat capacity anomaly intersection scenarios (with the density anomaly) would change in an analogous manner on swapping the TMD and TminD. Finally, we note that alternative definitions of compressibility anomaly (for example, taken at constant density as considered in Refs. 61 and 70) naturally follow the pattern of a second order anomaly as defined here.

\section{CONCLUSION}

In this paper, we have considered the origin of, and interactions between, a range of key thermodynamic anomalies and also the interactions with the stability limits. The conditions for these interactions have been derived from the underlying thermodynamic relations rather than using the more-commonly applied Taylor expansion method. We have also considered the behavior of the anomalies in terms of previously constructed thermodynamic "scenarios." All of the derived equations and interactions are universal in the sense they are valid for any single-component liquid and should apply to any potential satisfying this condition. We have tested the robustness of our derivations by comparing them to the results of numerical simulations and previous Taylor expansion-based results. Molecular dynamic simulations were performed using a modified Stillinger-Weber potential in which the balance of the two- and three-body interactions was varied. Employing replica exchange methods allowed us to extend the simulations into the deeply supercooled regime. This produces two types of behavior: in the first, the density anomalies collide with the stability limit, whilst in the second, they avoid collision and form complete thermodynamic loops. Finally, our more complete picture of the interactions of the anomalies allows us to propose a classification strategy for the thermodynamic anomalies which could be readily extended.

\section{SUPPLEMENTARY MATERIAL}

See supplementary material for detailed derivations of certain equations and discussions regarding mathematical details of the derivations.

\section{ACKNOWLEDGMENTS}

D.F. is grateful to St. Edmund Hall, the Clarendon Fund (University of Oxford) and International Research Fellowship of Japan Society for the Promotion of Science (JSPS) for financial support. We are grateful for support from the EPSRC Centre for Doctoral training, Theory and Modelling in Chemical Sciences, under Grant No. EP/L015722/1. We are grateful to the UK Materials and Molecular Modelling Hub for computational resources, which are partially funded by EPSRC (Grant No. EP/P020194/1). This paper conforms to the RCUK data management requirements. 


\section{REFERENCES}

${ }^{1}$ S. Sastry, F. Sciortino, and H. E. Stanley, "Limits of stability of the liquid phase in a lattice model with water-like properties," J. Chem. Phys. 98, 9863-9872 (1993).

${ }^{2}$ P. G. Debenedetti, "Supercooled and glassy water," J. Phys.: Condens. Matter 15, R1669-R1726 (2003); e-print arXiv:1708.02002.

${ }^{3}$ P. H. Poole, F. Sciortino, U. Essmann, and H. E. Stanley, "Phase behaviour of metastable water," Nature 360, 324-328 (1992).

${ }^{4}$ S. Sastry, P. G. Debenedetti, F. Sciortino, and H. E. Stanley, "Singularity-free interpretation of the thermodynamics of supercooled water," Phys. Rev. E 53, 6144-6154 (1996).

${ }^{5}$ J. R. Errington and P. G. Debenedetti, "Relationship between structural order and the anomalies of liquid water," Nature 409, 318-321 (2001).

${ }^{6}$ M. S. Shell, P. G. Debenedetti, and A. Z. Panagiotopoulos, "Molecular structural order and anomalies in liquid silica," Phys. Rev. E: Stat. Phys., Plasmas, Fluids, Relat. Interdiscip. Top. 66, 011202 (2002); e-print arXiv:0203383 [cond-mat].

${ }^{7}$ I. Saika-Voivod, F. Sciortino, and P. H. Poole, "Computer simulations of liquid silica: Equation of state and liquid-liquid phase transition," Phys. Rev. E 63, 011202 (2000); e-print arXiv:0007380 [cond-mat].

${ }^{8}$ M. Agarwal, R. Sharma, and C. Chakravarty, "Ionic melts with waterlike anomalies: Thermodynamic properties of liquid BeF2," J. Chem. Phys. 127, 164502 (2007).

${ }^{9}$ M. Agarwal and C. Chakravarty, "Waterlike structural and excess entropy anomalies in liquid beryllium fluoride," J. Phys. Chem. B 111, 13294-13300 (2007).

${ }^{10}$ B. S. Jabes, M. Agarwal, and C. Chakravarty, "Tetrahedral order, pair correlation entropy, and waterlike liquid state anomalies: Comparison of $\mathrm{GeO}_{2}$ with $\mathrm{BeF}_{2}$, $\mathrm{SiO}_{2}$, and $\mathrm{H}_{2} \mathrm{O}$," J. Chem. Phys. 132, 234507 (2010).

${ }^{11}$ B. Shadrack Jabes, D. Nayar, D. Dhabal, V. Molinero, and C. Chakravarty, "Water and other tetrahedral liquids: Order, anomalies and solvation," J. Phys.: Condens. Matter 24, 284116 (2012).

${ }^{12}$ G. S. Kell, "Density, thermal expansivity, and compressibility of liquid water from $0^{\circ}$ to $150^{\circ} \mathrm{C}$ : Correlations and tables for atmospheric pressure and saturation reviewed and expressed on 1968 temperature scale," J. Chem. Eng. Data 20, 97-105 (1975).

${ }^{13}$ C. A. Angell and H. Kanno, "Density maxima in high-pressure supercooled water and liquid silicon dioxide," Science 193, 1121-1122 (1976).

${ }^{14}$ C. A. Angell, R. D. Bressel, M. Hemmati, E. J. Sare, and J. C. Tucker, "Water and its anomalies in perspective: Tetrahedral liquids with and without liquid-liquid phase transitions," Phys. Chem. Chem. Phys. 2, 1559-1566 (2000).

${ }^{15}$ F. Caupin, "Escaping the no man's land: Recent experiments on metastable liquid water," J. Non-Cryst. Solids 407, 441-448 (2015).

${ }^{16}$ V. Holten, C. E. Bertrand, M. A. Anisimov, and J. V. Sengers, "Thermodynamics of supercooled water," J. Chem. Phys. 136, 094507 (2012).

${ }^{17}$ D. E. Hare and C. M. Sorensen, "The density of supercooled water. II. Bulk samples cooled to the homogeneous nucleation limit," J. Chem. Phys. 87, 4840-4845 (1987).

${ }^{18}$ L. B. Skinner, C. J. Benmore, J. C. Neuefeind, and J. B. Parise, "The structure of water around the compressibility minimum," J. Chem. Phys. 141, 214507 (2014).

${ }^{19}$ L. I. Aptekar, "Phase transitions in noncrystalline germanium and silicon," Phys.-Dokl. 24, 993 (1979).

${ }^{20} \mathrm{H}$. Thurn and J. Ruska, "Change of bonding system in liquid SexTe1-1 alloys as shown by density measurements," J. Non-Cryst. Solids 22, 331-343 (1976).

${ }^{21}$ S. J. Kennedy and J. C. Wheeler, "On the density anomaly in sulfur at the polymerization transition,” J. Chem. Phys. 78, 1523-1527 (1983).

${ }^{22} \mathrm{Y}$. Tsuchiya, "The anomalous negative thermal expansion and the compressibility maximum of molten Ge-Te alloys,” J. Phys. Soc. Jpn. 60, 227-234 (1991).

${ }^{23}$ P. Poole, M. Hemmati, and C. Angell, "Comparison of thermodynamic properties of simulated liquid silica and water," Phys. Rev. Lett. 79, 2281-2284 (1997).

${ }^{24}$ E. Lascaris, M. Hemmati, S. V. Buldyrev, H. E. Stanley, and C. A. Angell, "Search for a liquid-liquid critical point in models of silica," J. Chem. Phys. 140, 224502 (2014); e-print arXiv:1402.4452.
${ }^{25}$ P. A. Apte, N. Pingua, A. K. Gautam, U. Kumar, S. Y. Willow, X. C. Zeng, and B. D. Kulkarni, "The freezing tendency towards 4-coordinated amorphous networks causes an increase in the heat capacity of supercooled Stillinger-Weber silicon," RSC Adv. 5, 44679-44686 (2015).

${ }^{26}$ R. K. Endo, Y. Fujihara, and M. Susa, "Calculation of the density and heat capacity of silicon by molecular dynamics simulation," High Temp.-High Pressures 35-36, 505-511 (2003).

${ }^{27}$ G. Zhao, J. L. Yan, Y. J. Yu, M. C. Ding, X. G. Zhao, and H. Y. Wang, "Relationship between structural order and water-like anomalies in metastable liquid silicon: Ab initio molecular dynamics," Sci. Rep. 7, 39952 (2017).

${ }^{28}$ V. V. Vasisht, J. Mathew, S. Sengupta, and S. Sastry, "Nesting of thermodynamic, structural, and dynamic anomalies in liquid silicon," J. Chem. Phys. 141, 124501 (2014).

${ }^{29}$ V. V. Vasisht, S. Saw, and S. Sastry, "Liquid-liquid critical point in supercooled silicon," Nat. Phys. 7, 549-553 (2011).

${ }^{30} \mathrm{D}$. Fijan and M. Wilson, "Liquid state anomalies and the relationship to the crystalline phase diagram," Phys. Rev. E 99, 010103 (2019).

${ }^{31}$ D. Dhabal, C. Chakravarty, V. Molinero, and H. K. Kashyap, "Comparison of liquid-state anomalies in Stillinger-Weber models of water, silicon, and germanium," J. Chem. Phys. 145, 214502 (2016).

${ }^{32}$ J. Russo, K. Akahane, and H. Tanaka, "Water-like anomalies as a function of tetrahedrality," Proc. Natl. Acad. Sci. U. S. A. 115, E3333 (2018); e-print arXiv:1408.1149.

${ }^{33}$ D. Dhabal, A. H. Nguyen, M. Singh, P. Khatua, V. Molinero, S. Bandyopadhyay, and C. Chakravarty, "Excess entropy and crystallization in Stillinger-Weber and Lennard-Jones fluids," J. Chem. Phys. 143, 164512 (2015).

${ }^{34}$ W. Hujo, B. S. Jabes, V. K. Rana, C. Chakravarty, and V. Molinero, "The rise and fall of anomalies in tetrahedral liquids," J. Stat. Phys. 145, 293-312 (2011); e-print arXiv:1107.5623.

${ }^{35}$ S. Sengupta, V. V. Vasisht, and S. Sastry, "Diffusivity anomaly in modified Stillinger-Weber liquids," J. Chem. Phys. 140, 044503 (2014).

${ }^{36}$ F. H. Stillinger and T. A. Weber, "Computer simulation of local order in condensed phases of silicon,” Phys. Rev. B 31, 5262-5271 (1985).

${ }^{37}$ E. B. Moore and V. Molinero, "Growing correlation length in supercooled water," J. Chem. Phys. 130, 244505 (2009).

${ }^{38}$ M. H. Bhat, V. Molinero, E. Soignard, V. C. Solomon, S. Sastry, J. L. Yarger, and C. A. Angell, "Vitrification of a monatomic metallic liquid," Nature 448, 787-790 (2007).

${ }^{39} \mathrm{~V}$. Molinero and E. B. Moore, "Water modeled as an intermediate element between carbon and silicon," J. Phys. Chem. B 113, 4008-4016 (2009).

${ }^{40}$ A. S. Barnard and S. P. Russo, "Development of an improved StillingerWeber potential for tetrahedral carbon using ab initio (Hartree-Fock and MP2) methods," Mol. Phys. 100, 1517-1525 (2002).

${ }^{41}$ P. C. Hemmer and G. Stell, "Fluids with several phase transitions," Phys. Rev. Lett. 24, 1284-1287 (1970).

${ }^{42}$ E. A. Jagla, "Minimum energy configurations of repelling particles in two dimensions," J. Chem. Phys. 110, 451 (1998).

${ }^{43}$ M. A. A. Barbosa, F. V. Barbosa, and F. A. Oliveira, "Thermodynamic and dynamic anomalies in a one-dimensional lattice model of liquid water," J. Chem. Phys. 134, 024511 (2011).

${ }^{44}$ E. O. Rizzatti, M. A. A. Barbosa, and M. C. Barbosa, "Core-softened potentials, multiple liquid-liquid critical points, and density anomaly regions: An exact solution," Front. Phys. 13, 136102 (2018).

${ }^{45}$ F. B. V. da Silva, F. A. Oliveira, and M. A. A. Barbosa, "Residual entropy and waterlike anomalies in the repulsive one dimensional lattice gas," J. Chem. Phys. 142, 144506 (2015).

${ }^{46} \mathrm{~J}$. R. Bordin and M. C. Barbosa, "Waterlike anomalies in a two-dimensional core-softened potential," Phys. Rev. E 97, 022604 (2018).

${ }^{47}$ M. A. A. Barbosa, E. Salcedo, and M. C. Barbosa, "Multiple liquid-liquid critical points and density anomaly in core-softened potentials," Phys. Rev. E 87, 032303 (2013).

${ }^{48} \mathrm{E}$. A. Jagla, "Core-softened potentials and the anomalous properties of water," J. Chem. Phys. 111, 8980 (1999). 
${ }^{49}$ N. M. Barraz, E. Salcedo, and M. C. Barbosa, "Thermodynamic, dynamic, and structural anomalies for shoulderlike potentials," J. Chem. Phys. 131, 094504 (2009).

${ }^{50}$ A. Barros de Oliveira, P. A. Netz, T. Colla, and M. C. Barbosa, "Thermodynamic and dynamic anomalies for a three-dimensional isotropic core-softened potential," J. Chem. Phys. 124, 084505 (2006).

${ }^{51}$ N. M. Barraz, E. Salcedo, and M. C. Barbosa, "Thermodynamic, dynamic, structural, and excess entropy anomalies for core-softened potentials," J. Chem. Phys. 135, 104507 (2011); e-print arXiv: 1106.5069.

${ }^{52}$ J. N. Da Silva, E. Salcedo, A. B. De Oliveira, and M. C. Barbosa, "Effects of the attractive interactions in the thermodynamic, dynamic, and structural anomalies of a two length scale potential," J. Chem. Phys. 133, 244506 (2010); e-print arXiv: $1006.0033 \mathrm{v} 1$.

${ }^{53}$ R. J. Speedy, "Stability-limit Conjecture. An interpretation of the properties of water," J. Phys. Chem. 86, 982-991 (1982).

${ }^{54} \mathrm{R}$. J. Speedy, "Limiting forms of the thermodynamic divergences at the conjectured stability limits in superheated and supercooled water," J. Phys. Chem. 86, 3002-3005 (1982).

${ }^{55}$ P. G. Debenedetti and M. C. D'Antonio, "On the nature of the tensile instability in metastable liquids and its relationship to density anomalies," J. Chem. Phys. 84, 3339-3345 (1986).

${ }^{56}$ P. G. Debenedetti and M. C. D'Antonio, "On the entropy changes and fluctuations occurring near a tensile instability," J. Chem. Phys. 85, 4005-4010 (1986).

${ }^{57}$ M. C. D'Antonio and P. G. Debenedetti, "Loss of tensile strength in liquids without property discontinuities: A thermodynamic analysis," J. Chem. Phys. 86, 2229-2235 (1987).

${ }^{58}$ P. G. Debenedetti and M. C. D'Antonio, "Stability and tensile strength of liquids exhibiting density maxima," AIChE J. 34, 447-455 (1988).
${ }^{59}$ L. P. N. Rebelo, P. G. Debenedetti, and S. Sastry, "Singularity-free interpretation of the thermodynamics of supercooled water. II. Thermal and volumetric behavior," J. Chem. Phys. 109, 626-633 (1998).

${ }^{60}$ P. H. Poole, I. Saika-Voivod, and F. Sciortino, "Density minimum and liquidliquid phase transition," J. Phys.: Condens. Matter 17, L431 (2005).

${ }^{61}$ V. Holten, C. Qiu, E. Guillerm, M. Wilke, J. Rička, M. Frenz, and F. Caupin, "Compressibility anomalies in stretched water and their interplay with density anomalies," J. Phys. Chem. Lett. 8, 5519-5522 (2017); e-print arXiv:1708.00063.

${ }^{62}$ Y. Sugita and Y. Okamoto, "Replica-exchange molecular dynamics method for protein folding,” Chem. Phys. Lett. 314, 141-151 (1999).

${ }^{63} \mathrm{R}$. Swendsen and J. Wang, "Replica Monte Carlo simulation of spin glasses," Phys. Rev. Lett. 57, 2607-2609 (1986).

${ }^{64}$ L. Rovigatti, V. Bianco, J. M. Tavares, and F. Sciortino, "Communication: Reentrant limits of stability of the liquid phase and the Speedy scenario in colloidal model systems," J. Chem. Phys. 146, 041103 (2017).

${ }^{65}$ V. P. Skripov, "Important property of a spinodal curve," Teplofiz. Vys. Temp. 4, 816-820 (1966)

${ }^{66}$ S. Plimpton, "Fast parallel algorithms for short-range molecular dynamics," J. Comput. Phys. 117, 1-19 (1995)

${ }^{67}$ S. Nosé, "A unified formulation of the constant temperature molecular dynamics methods," J. Chem. Phys. 81, 511-519 (1984).

${ }^{68}$ W. G. Hoover, "Canonical dynamics: Equilibrium phase-space distributions," Phys. Rev. A 31, 1695-1697 (1985).

${ }^{69}$ L. S. Caves, J. D. Evanseck, and M. Karplus, "Locally accessible conformations of proteins: Multiple molecular dynamics simulations of crambin," Protein Sci. 7, 649-666 (1998)

${ }^{70}$ Y. E. Altabet, R. S. Singh, F. H. Stillinger, and P. G. Debenedetti, “Thermodynamic anomalies in stretched water," Langmuir 33, 11771 (2017). 